\title{
THEORETICAL STUDY OF THERMOGALVANIC CELLS IN STEADY STATE
}

\author{
Artjom V. SOKIRKO* \\ Department of Hydromechanics, The Royal Institute of Technology, 10044 Stockholm, Sweden
}

(Received 15 February 1993; in revised form 23 September 1993)

\begin{abstract}
The functioning of thermogalvanic cells has been studied theoretically. The analysis for the simplest case which accounts for only diffusion transport of the reacting substances from one electrode to another has been made. The simple relation for thermodynamic efficiency of such energy sources was obtained. The diffusion-migration transport for the horizontally arranged cell and the diffusionconvection transport for the vertically arranged cell were studied in detail. The original mathematical methods for solving transport equations were used. Analytical results for the concentration distribution and the current-voltage curves were calculated. The principal role of electrode kinetics (fast changing of the equilibrium electrode potential with the temperature) was shown. Finally, the general algorithm for improving thermogalvanic cells was proposed.
\end{abstract}

Key words: diffusion-migration transport, fluid motion, efficiency concentration distribution, currentvoltage curve.

\section{NOMENCLATURE}

$a, b$

$A i$

$\hat{c}$

$c^{0}$

$c_{\mathrm{i}}$

$c_{3}$

$\tilde{c}_{\mathbf{k}}$

$c_{\mathrm{i}}^{0}$

$\left.c_{\mathrm{i}}\right|_{x_{\mathrm{L}}},\left.c_{\mathrm{i}}\right|_{x_{\mathrm{R}}}$

$\Delta c$

$D_{\mathrm{k}}$

$D_{\mathbf{k}}^{\mathrm{T}}$

$e$

$F$

$F_{m}^{(l)}$

$G_{l j}$

${ }^{*}$ Permanent address: A. N. Frumkin Institute of Electrochemistry, Russian Academy of Sciences, Leninsky Prospect 31, 117071 Moscow, Russia. function, corresponding to the boundary conditions

Airy function specific thermal capacity $/ \mathrm{J} \mathrm{kg}^{-1} \mathrm{~K}^{-1}$ initial concentration of salt $/ \mathrm{mol} \mathrm{m}^{-3}$ concentrations of cations species $\mathbf{i}$,/ $\mathrm{mol} \mathrm{m}^{-3}$

anions concentration $/ \mathrm{mol} \mathrm{m}^{-}$

dimensionless concentrations of ions species $\mathrm{k}(\mathrm{k}=1,2,3)$

initial concentrations of ions species $\mathrm{i} / \mathrm{mol} \mathrm{m}^{-3}$

concentrations of ions species $i$ at the electrode surfaces $/ \mathrm{mol} \mathrm{m}^{-3}$

difference of the total concentration/ $\mathrm{mol} \mathrm{m}^{-3}$

diffusion coefficients of the $\mathrm{k}$-th ion

$(\mathrm{k}=1,2,3) / \mathrm{m}^{2} \mathbf{s}^{-1}$

thermal diffusion coefficient of the

mol m ${ }^{-1} \mathbf{s}^{-1} \mathbf{K}^{-1}$

$=2.71828$

ort in the direction of axis y

index of adding

Faraday's constant/96487 $\mathrm{C} \mathrm{mol}^{-1}$ coefficients of Fourier series for cations of kind $m$

gravitation constant $/ 9.81 \mathrm{~m} \mathrm{~s}^{-2}$

matrix of a linear system

cell height $/ \mathrm{m}$

imaginary unity

electrical current/A $\mathrm{k}$-th ion $(\mathrm{k}=1,2,3) /$
$\underline{I_{0}}$

$\bar{I}$

$j_{0}$

$j_{1}, j_{2}$

$\tilde{j}$

\section{$J$}

$J_{\mathrm{c}}$

$J_{h}$

$J_{\mathrm{h}}^{\prime}$

$k_{1}, k_{2}$

K

$l$

$L$

$M$

$\mathbf{M}^{2_{1}+}, \mathbf{M}^{2_{2}+}$

$\dot{n}$

$N$

$p$

$P$

$P_{\max }$

$Q_{\mathrm{f}}$

$r$

$r_{0}$

$r_{\text {ext }}$

$\operatorname{Re}$

S

$S$

$T$

$T_{0}$

$T_{\mathrm{i}}$ current of short circuit/A

integral current/A

density of electric current/ $\mathrm{A} \mathrm{m}^{-2}$

exchange current density/ $\mathrm{A} \mathrm{m}^{-2}$

current densities, transported by ion species 1 and $2 / \mathrm{A} \mathrm{m}^{-2}$

dimensionless current density

renormed dimensionless current density

maximum density of electric power/ $\mathrm{W} \mathrm{m} \mathrm{m}^{-2}$

thermal density flux/ $\mathrm{W} \mathrm{m}^{-2}$

transported entropy/W $\mathbf{m}^{-2}$

rate constants of forward and back reactions, respectively

ratio of initial concentration

index of adding

distance between electrodes $/ \mathrm{m}$

constant of integration

ions of the redox couple

axis, directed from the electrode into the solution

constant of integration

pressure/Pa

useful power/W

maximal useful power/W

vector for linear system

internal resistance $\mathbf{\Omega}$

internal resistance a open circuit $\mathbf{\Omega}$

external resistance/ $\mathbf{\Omega}$

universal gas constant/

$8.314 \mathrm{~J} \mathrm{~mol}^{-1} \mathrm{~K}^{-1}$

Reynolds number

constant of integration

area of electrode $/ \mathrm{m}^{2}$

absolute temperature/K

average values of temperature/K

temperature of electrodes $(i=1,2) / K$ 


\section{$\boldsymbol{u}_{\mathbf{k}} \quad$ mobility of the $\mathrm{k}$-th ion $(\mathrm{k}=1,2,3)$ / \\ $U$ \\ $U_{0}$ \\ $\boldsymbol{v}$ \\ $w_{1}, w_{2}$ \\ $x$ \\ $x_{\mathbf{L}}, x_{\mathbf{R}}$ \\ $\tilde{\boldsymbol{x}}$ \\ $y$ \\ $z_{1}, z_{2}$ \\ $z_{3}$ \\ $Z_{1}, Z_{2}$ \\ $\alpha$ \\ $\beta$ \\ $\bar{\beta}$ \\ $\gamma_{\mathrm{i}}$ \\ $\eta$ \\ $\eta_{\text {Carnot }}$ \\ $\eta_{\text {real }}$ \\ $\theta_{m}$ \\ $\Theta$

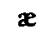 \\ $\mathrm{m}^{2} \mathrm{~s}^{-1} \mathrm{v}^{-1}$ \\ cell potential/V \\ cell potential at open circuit $-\mathrm{V}$ \\ fluid velocity $/ \mathrm{ms}^{-1}$ \\ vector of the right part of linear system \\ intermediate parameter of theory \\ coordinate, directed from one elec- trode to another $/ \mathrm{m}$ \\ coordinates of electrodes $/ \mathrm{m}$ \\ dimensionless coordinate \\ coordinate, directed along the elec- trode surface/m \\ charge numbers of cations \\ charge number of anions \\ ratios of charge numbers \\ transfer coefficient \\ thermal expansion coefficient $/ \mathrm{m}^{3} \mathrm{~K}^{-1}$ coefficient of dependence of the density on concentration $/ \mathrm{m}^{3} \mathrm{~mol}^{-1}$ \\ combinations of charge numbers and diffusion coefficients \\ normalised efficiency \\ efficiency of the ideal thermodynami- cal system \\ efficiency of the real thermodynami- cal system \\ dimensionless parameter $\boldsymbol{\Theta}, \boldsymbol{m}=1,2$ \\ parameter of theory $/ \mathrm{m}^{-1}$ \\ specific resistance $/ \mathbf{\Omega} \mathrm{m}$}

$\kappa$

$\hat{\boldsymbol{\kappa}}$

$\lambda^{0}$

$\Lambda$

$\mu, \mu$

$v$

$\xi$

$\rho$

$\sigma$

$\dot{\tau}$

$\phi$

$\Phi$

$\chi$

$\psi_{1}, \psi_{2}$

$\omega$

temperature conductivity coefficient/ $\mathrm{m}^{2} \mathrm{~s}^{-1}$

coefficient of thermal conductivity of liquid/W $\mathrm{m}^{-1} \mathrm{~K}^{-1}$

equivalent electroconductivity/

$\Omega \mathrm{m}^{4} \mathrm{~mol}^{-1}$

dimensionless combination of diffusion coefficients

value of Airy function and its derivatives at zero

kinetic viscosity $/ \mathrm{m}^{2} \mathrm{~s}^{-1}$

surface overpotential/V

density of liquid/ $\mathrm{Kg} \mathrm{m}^{-3}$

dimensionless exchange current density

coordinate, directed along the surface of the electrodes

potential/V

equilibrium potential/V

stream function $/ \mathrm{m}$

ratio of diffusion current density and exchange current density dimensionless potential

values of a dimensionless potential at the electrodes

intermediate parameter

\section{INTRODUCTION}

Direct transformation of thermal energy into electrical by means of thermogalvanic cells (TGC) on an electrochemical basis is one of the most attractive ways of energetics development. The characteristic features of those systems, such as the absence of any moving mechanical parts, the absolutely complete and closed usage of solutions, longevity and simplicity of use are their obvious merits [1-4]. The fundamentals of thermogalvanic cells are discussed in $[5$, 6].

Such research is carried out most actively in Japan due to significant amounts of geothermal water supplies. That research includes experimental studies of the functioning of different TGC variants, as well as model calculations by supercomputers [7, 8]. The main shortcoming of TGCs is their extremely low efficiency that makes their application in common practice impossible. The main aim of researchers of TGC is to find, experimentally the characteristics of the device (the temperature of electrodes, contents, geometrical size), which would provide the highest efficiency. Model calculations of such systems demand significant computer time. This prevents the systematic research of all possible variants of TGC. Actually, only a few calculations for some variants of TGC, more or less related to those real systems that already exist, have been made [9, 10]. This is why it is necessary to work out a reasonably simple theoretical model which can help in studies of the principal properties of TGC and also provide a theoretical estimation of the efficiency of present TGC constructions.

The first three parts of the present paper are devoted to general properties of TGC and also to the qualitative estimation of their efficiency. The main steps of obtaining the current-voltage curves for TGC are described in the fourth part. It allows us to obtain a more accurate evaluation of the efficiency. The final two parts are devoted to the discussion of the fluid flow in the volume of TGC and to the distribution of concentrations arising under the diffusion and convection effects.

\section{TGC as an electrical system}

TGC is the usual source of current (Fig. 1). The effective voltage, as well as the electric current in the system varies with the external resistance $\boldsymbol{r}_{\text {ext }}$. The qualitative behaviour of $I\left(r_{\text {ex }}\right)$ and $U\left(r_{\text {ext }}\right)$ dependencies is shown in Fig. 2. When $r_{\text {ext }} \rightarrow 0$, the current $I$ tends to $I_{0}$ (the current of the short circuit) and

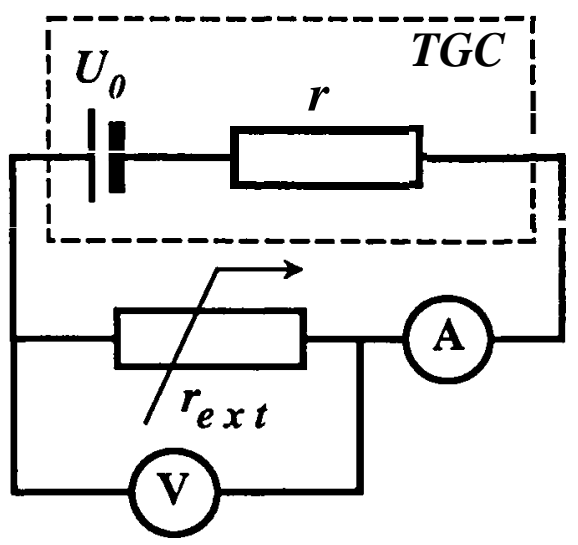

Fig. 1. The principal electrical scheme of a TGC. 

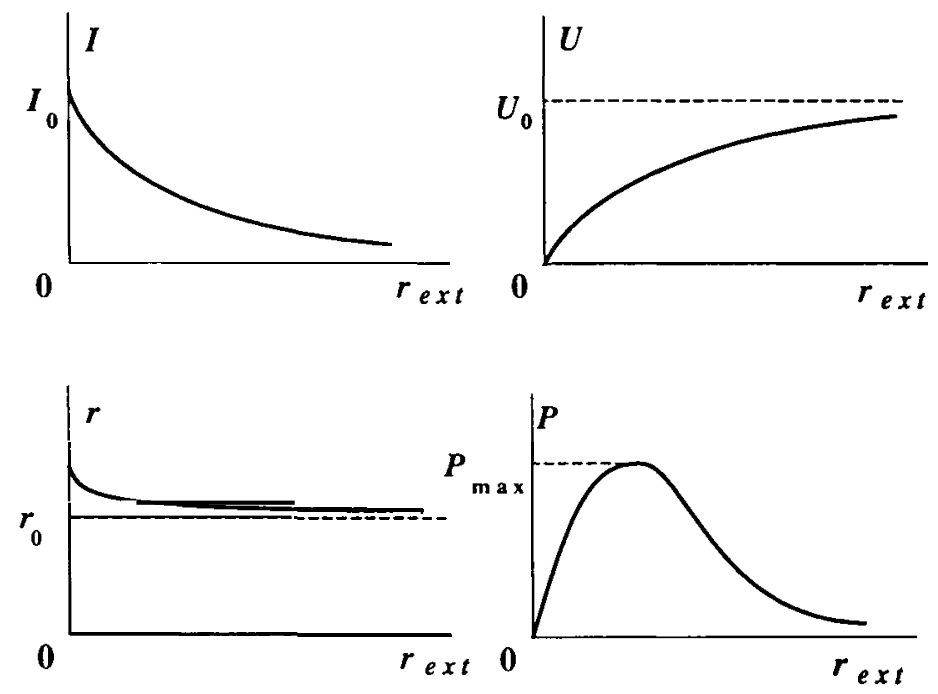

Fig. 2. The typical dependences of the current $I$, the effective voltage $U$, the internal resistance $r$ and the useful power from the external resistance $r_{\text {ext }}$.

when $\boldsymbol{r}_{\text {ext }} \rightarrow \infty$ and $\mathrm{I} \rightarrow 0$, the voltage on the load tends to $U_{0}$, the open-circuit voltage. In the general case, the inner resistance $\boldsymbol{r}$ can be calculated from the second Kirchhoff relationship for the closed circuit: $\boldsymbol{r}=U_{0} / I-r_{\text {ext }}: \mathrm{r}$ is a function of $\boldsymbol{r}_{\text {ext }}$, as well as of useful power $\mathrm{P}=\boldsymbol{U} I$ educed on $\boldsymbol{r}_{\mathrm{ext}}$ (Fig. 2). But over the main range of $\boldsymbol{r}_{\mathbf{e x t}}$ the function $\boldsymbol{r}\left(\boldsymbol{r}_{\mathbf{e x}}\right)$ is almost constant, equal to the internal resistance $r_{0}$ of the open circuit.

It can be easily seen that power $P_{\max }$, educed on the external resistance, becomes maximal under the condition of equality of inner and external loads $r_{0} \approx r_{\mathrm{ext}}$ and is equal to

$$
P_{\max } \approx \frac{U_{0}^{2}}{4 r_{0}} .
$$

The same power, obviously is reduced on the internal resistance $\boldsymbol{r}_{\mathbf{0}}$.

The most important difference of TGC from usual electrochemical sources of current is that the useful capacity is produced at the expense of a temperature difference between electrodes and does not depend on a power educed on the inner resistance. Even in the open-circuit case, almost the same energy is required for maintaining the temperature difference between electrodes, as for working in the optimum regime. That is why $P_{\max }$, given by formula (1), is the optimum value. Thus, to avoid the additional losses of power, the electrical resistance in the external device should be always constant and equal to the internal resistance of TGC.

Some experimental current-voltage dependencies for TGC are given in [7]. They look typical for electrochemical systems, ie the current reaches limiting values in the case of sufficiently high modules of potential difference. The only difference is that this curve does not pass through the origin $\boldsymbol{U}=0, \mathrm{I}=0$. The point of intersection of this curve with the axis $\boldsymbol{U}\left(\boldsymbol{U}=\boldsymbol{U}_{\mathbf{0}}, \mathbf{I}=0\right)$ relates to the completely open circuit, the point of intersection with the axis $I(U=0, I=I$,$) relates to the short circuit. These$ two points are situated not far from each other (in comparison with the characteristic value of the limiting current in the system) and get closer when the temperature difference is lowered. Obviously, a certain regime of TGC functioning, ie a certain value of $\boldsymbol{r}_{\text {ext }}$, correlates to each point of the curve segment between $\boldsymbol{U}_{\mathbf{0}}$ and I,. The points outside this segment can principally not be reached when the TGC is used as the current source. It means that the real TGC functions in a regime far from limiting one (near the equilibrium) when the current-voltage curve can be considered an approximately straight line, which does not pass the zero point.

\section{Dimensional analysis of TGCfunctioning as a thermodynamic system}

Let us consider the simplest TGC model, consisting of two large parallel plates placed a small (in comparison with their size) distance $\mathrm{L}$ from each other and heated up to different temperature values $T_{1}$ and $T_{2}$. Then, the thermal density flux $J$, from the heated plate to the cold one can be calculated as

$$
J_{\mathrm{b}}=\hat{\kappa} \frac{T_{2}-T_{1}}{L}
$$

where $\hat{\kappa}$ is the coefficient of thermal conductivity of liquid between the plates. In equation (2) we presumed that there was no convective flow of solution. Sometimes, instead of the thermal conductivity coefficient $\hat{\kappa}$, it is more convenient to use the temperature conductivity coefficient: $\mathrm{K}=\hat{\boldsymbol{\kappa}} / \hat{\boldsymbol{c}} \boldsymbol{\rho}$, where $\mathrm{p}$ is the density of the liquid and $\hat{c}$ is the specific thermal capacity. The heat absorption [5] due to the entropy change of the electrode reaction and the transported entropy $J_{h}^{\prime}$ is proportional to the temperature, current density and thermoelectric power of the system $U_{0} /\left(T_{2}-T_{1}\right)$

$$
J_{h}=\frac{I}{S} T_{2} \frac{U_{0}}{T_{2}-T_{1}},
$$


where $\mathrm{S}$ is the area plates. The heat supplied to the system is $J_{h}+J_{\mathbf{h}}^{\prime}$.

The maximum density of the electric power $\boldsymbol{J}_{\boldsymbol{c}}$ can be calculated as $J_{\mathbf{e}}=P_{\mathrm{ma}} J S$. The inner resistance of TGC, $\boldsymbol{r}_{\mathbf{0}}$, can be presented as $\boldsymbol{r}_{\mathbf{0}}=\boldsymbol{x} \boldsymbol{L} / \boldsymbol{S}$ where $\boldsymbol{x}$ is the specific resistance and can be evaluated as $£=$ $\left(c^{0} \lambda^{0}\right)^{-1}$, where $c^{0}$ being the concentration of salt, $\lambda^{0}$ is the equivalent electroconductivity. Thus, the electric power density can be presented as

$$
J_{\mathrm{e}}=\frac{U_{0}^{2} c^{0} \lambda^{0}}{4 L}
$$

It is more convenient to use the normalised efficiency $\boldsymbol{\eta}=\boldsymbol{\eta}_{\text {real }} / \boldsymbol{\eta}_{\text {Carnat }}$ which is the ratio of efficiency of real engines $\eta_{\text {real }}=J_{\mathrm{e}} / J_{\mathrm{h}}+J_{\mathrm{h}}^{\prime}$ to $\eta_{\text {Carnot }}=T_{2}-T_{1} / T_{2}$ - the efficiency of the ideal thermodynamical system, functioning by Carnot cycle. All efficiencies $\left(\boldsymbol{\eta}_{\text {real }}\right.$, $\eta_{\text {Carnot }}$ and $\boldsymbol{\eta}$ ) are less than unity:

$$
\eta=\left\{1+\left(\frac{T_{2}-T_{1}}{U_{0}}\right)^{2} \frac{4 \hat{\kappa}}{T_{2} c^{0} \lambda^{0}}\right\}^{-1} .
$$

We can call the thermodynamic system "very good" if $\boldsymbol{\eta}$ about 1 and "very bad" if $\boldsymbol{\eta} \approx \mathbf{0}$. The value of the efficiency itself is not as readily meaningful as the value of the norrnalised efficiency. Therefore, exactly normalised efficiency $\boldsymbol{\eta}$ can be abbreviated to "efficiency" in the ordinary sense.

The only dependent parameter, $\boldsymbol{U}_{0}$, involved in the equation (4), can be obtained from the analysis of TGC electrochemical kinetics.

\section{Electrochemical model of TGC functioning}

The real TGC is an electrochemical system with a concentrated mixture of two salts, for example $\mathrm{FeCl}_{2}$ and $\mathrm{FeCl}_{3}$, placed between two identical unconsummated electrodes. The same electrochemical reaction takes place on both the electrodes:

$$
\mathrm{M}^{z_{1}+}+\left(z_{1}-z_{2}\right) e^{-} \underset{k_{2}}{\stackrel{k_{1}}{\rightleftarrows}} \mathbf{M}^{z_{2}+},
$$

where $\boldsymbol{z}_{\mathbf{1}}$ and $\boldsymbol{z}_{\mathbf{2}}$ are the charge numbers of cations. Rate constants of forward $(\mathrm{k}$,$) and back \left(k_{2}\right)$ reactions depend on the temperature and that is why they are different for different electrodes. Equilibrium concentrations, defined by the ratio $k_{1} / k_{2}$, appear different at different electrodes. It causes ion transport from one electrode towards another, and provides for the reaction process in the anodic direction on one electrode, and in cathodic direction on the other one.

In accordance with generally accepted designations[11], density of the electric current, flowing through the surface of the electrode, can be presented as follows [12] :

$$
\begin{array}{r}
j=j_{0}\left(\frac{\left.c_{1}\right|_{\mathrm{s}}}{c_{1}^{0}} \exp \left(\frac{-\left(z_{1}-z_{2}\right)(1-\alpha) F \xi}{R T}\right)\right. \\
\left.-\frac{\left.c_{2}\right|_{\mathrm{s}}}{c_{2}^{0}} \exp \left(\frac{\left(z_{1}-z_{2}\right) \alpha F \xi}{R T}\right)\right),
\end{array}
$$

where $\boldsymbol{j}_{\mathbf{0}}$ is the exchange current density, $\boldsymbol{c}, \mathbf{l}_{\mathbf{s}}$ and $c_{2} \mathbf{l}_{\mathbf{s}}$ are concentrations of ions $\mathbf{M}^{\mathbf{z}_{1}+}$ and $\mathbf{M}^{\mathbf{z}_{2}+}$ in the vicinity of the electrode, $\boldsymbol{c}_{\mathbf{1}}^{\mathbf{0}}$ and $\boldsymbol{c}_{\mathbf{2}}^{\mathbf{0}}$ are the corresponding initial concentrations, $\mathrm{T}$ is the absolute temperature in the vicinity of the electrode, $\mathrm{R}$ is the universal gas constant, a is the symmetry factor and $\xi$ is the surface overpotential:

$$
\xi=\phi-\phi^{(\mathbf{s})},
$$

$\phi$ is the potential drop between the nearest to the electrode zone and the electrode and $\phi^{(\mathbf{s})}$ is the equilibrium potential.

The most frequently used systems for studying of TGC are $\left[\mathrm{Fe}(\mathrm{CN})_{6}\right]^{3-} \mid\left[\mathrm{Fe}(\mathrm{CN})_{6}\right]^{4-}$ (standard potential $\phi^{(\mathbf{s})}=0.36 \mathrm{~V}$ at $\left.\mathrm{T}=298 \mathrm{~K}\right)$ and $\mathrm{Fe}^{2+} \mid \mathrm{Fe}^{3+}$ (standard potential $\phi^{(\mathbf{s})}=0.771 \mathrm{~V}$ at $\mathrm{T}=298 \mathrm{~K}$ ). But in definition (7), the equilibrium potential, measured at the real temperature of the electrode, is included instead of the standard potential, measured at the standard temperature.

On open circuit the electrical current $j$ equals zero, therefore the potential drop inside the solution depends on Soret diffusion only. As will be shown in the next section, the corresponding Soret potential tends to lower the existing potential difference $\boldsymbol{U}_{\mathbf{0}}$ between the electrodes but it is usually significantly lower than $U_{0}$. It means that the potential drop between the electrodes may be estimated to a first approximation as

$$
U_{0}=\left.\phi^{(s)}\right|_{T=T_{2}}-\left.\phi^{(s)}\right|_{T=T_{1}},
$$

where $T_{1}$ and $T_{2}$ are the electrodes temperature values.* Substituting $U_{0}$ into (4), we obtain

$$
\eta=\left\{1+\left(\frac{T_{2}-T_{1}}{\left.\phi^{(s)}\right|_{T=T_{2}}-\left.\phi^{(s)}\right|_{T=T_{1}}}\right)^{2} \frac{4 \kappa}{T_{2} c^{0} \lambda^{0}}\right\}^{-1} .
$$

Note that in most cases the value of $\mathbf{J}$, is much greater than that of $\boldsymbol{J}_{\mathbf{h}}^{\prime}$ and for the case of a small temperature difference between the electrodes $\left(T_{2}-\mathrm{T},\right) \ll T_{2}$ we obtain the simplified expression:

$$
\eta=\left(\frac{d \phi^{(s)}}{d T}\right)^{2}\left(\frac{T c^{0} \lambda^{0}}{4}\right)\left(\frac{1}{\kappa \hat{c} \rho}\right)
$$

In expression (9) we assumed that the equilibrium potential of the electrochemical reaction depends on the temperature only. Substituting the parameter value into (9) in case when the concentrations of $\mathrm{FeCl}_{2}$ and $\mathrm{FeCl}_{3}$ in the mixture are both equal to $1.0 \mathrm{M}$, we get the approximate estimation for $\eta \sim 10^{-4}$. which agrees well with the experimental data [7].

As follows from relation (9), the normalised efficiency of TGC is defined as a product of three terms.

- As it had been pointed out by referee, $\boldsymbol{\phi}^{(\mathbf{)})}$ is normally defined as a potential difference of the cell $\mathbf{P t}(\mathbf{T}) \mid \mathbf{M}^{\mathbf{x}_{2}+}$, $\mathbf{M}^{\mathbf{z}_{\mathbf{2}}+} \mid \boldsymbol{n} \boldsymbol{n} \mathbf{( T )}$ where $\mathbf{P t}$ and $\boldsymbol{n h e}$ electrodes are at the same temperature. Therefore, in the expressions above $\phi^{(s)}$ must be defined with respect to a hypothetical electrode, whose potential does not depend on temperature. However, it is more reasonable instead of a hypothetical electrode to use the real one at a mean temperature $T_{3}: T_{1}<T_{3}<T_{2}$, ie $\phi^{(0)}\left(T_{1}\right)$ is defined as a potential difference of the cell $\mathbf{P t}\left(T_{1}\right)\left|\mathbf{M}^{z_{1}+}, \mathbf{M}^{z_{2}+}\right|$ nhe $\left(T_{3}\right)$. It has been shown that for the small temperature difference $\left(T,-T_{1}\right) / T_{2} \ll 1$ the value of $U_{0}$, defined by equation (8) does not depend on $\boldsymbol{T}_{\mathbf{3}}$ in the first approximation. 
The first of them characterises the type of the electrochemical reaction. In order to increase $\eta$, the electrochemical reactions that have the strongest dependence upon the temperature, should be used, and the temperature range where $\left|\mathbf{d} \phi^{(\mathbf{s})} / d T\right|$ reaches its maximum should be chosen.

The second term mainly characterises the electric conductivity of a cell. As mentioned by the majority of research. solutions should be concentrated up to the maximum value. In this case, parameter $\lambda^{\mathbf{0}}$ is not independent, but is completely defined by the composition of solution, concentration and its temperature.* It is important to emphasise, that in the stationary case under analysis, electroconductivity of the solution cannot be increased by means of addition of any supporting electrolyte, because the electric current in the system is conducted only by ions $\mathbf{M}^{\mathbf{z}_{1}+}$ and $\mathbf{M}^{\mathbf{z}_{2}+}$. (There is an important difference between the stationary case, when an ion of a supporting electrolyte does not move and the nonstationary case with ac or during the period right after switching on. In the time dependent problem the electric current is transported by supporting electrolyte ions as well.) The average value of the absolute temperature $T$ in the second term of (9) is varying within narrow limits and cannot significantly change the normalised efficiency $\eta$; moreover, as was already mentioned above, $\mathrm{T}$ is to be chosen deliberately in order to maximise the first term.

The last term is inverted thermal conductivity of the solution, ie of the solvent. Its value is, actually, constant for all water solutions and only slightly depends on the temperature and concentrations (the variation is less than $5 \%$ ).

Besides, the analysis of the factors, upon which the normalised efficiency $\eta$ does not depend, is not less important. At first, the normalised efficiency $\eta$ does not depend on the temperature difference between the electrodes (if it exists), because the potential difference $U_{0}$ in (4) is proportional to (T, - T, ) with high accuracy. Secondly, the normalised efficiency $\eta$ depends slightly on all geometrical sizes of the system, in particular, on the distance between electrodes. Geometrical sizes influence on the appearance of the natural convection in the liquid. Convection promotes the transport of electroactive ions from one electrode to another and reduces electric resistance of the system. But, at the same time, convection increases the heat transport from one electrode to another. As long as the mass diffusion coefficient is lower than the temperature diffusivity there is a region where the convection transfer of the heat increases slower than the mass transfer. In detail, this case will be considered in sections 5 and

${ }^{*}$ Strictly speaking, concentration $\boldsymbol{c}^{\mathbf{0}}$ is not $\boldsymbol{g o o d}$ defined value, because solution within TGC is always mixture of two substance. Reaction (6) on the both electrodes procedure with the same rate, which means that composition of solution does not change with time. Therefore, if the concentration of one of cations species is more than another one, the excess becomes electropassive. Therefore, the $c^{0}$ really means the minimum concentrations of the cations. In practice one only uses the mixture of two salts with equal concentration $c^{0}$. For such solutions the integral parameter $\lambda^{0}$ also becomes well defined.
6. Only the minor dependence of $\eta$ on the convection (natural or artificial) was found.

\section{Transport in TGC without convection transport}

Let us consider TGC in the absence of convective flows. It can be either a horizontal cell with heated upper electrode or the one with heated lower electrode (under additional condition $\mathrm{Ra}<\boldsymbol{R} \boldsymbol{a}_{\text {criticas }}$; for the pure solvent the convection flow, as in Bernar cells, arises if $\mathrm{Ra}>\boldsymbol{R} \boldsymbol{a}_{\text {critical }}=1708$ ). Convection can also be suppressed by injection of a porous filler between the electrodes or of some other separators[13].

The distance between the electrodes is much less than their size, consequently, it may be assumed that the processes are described by onedimensional transport equations. The temperature distribution in this case is calculated trivially: it changes linearly from the value $\boldsymbol{T}_{1}$ on one electrode to $\boldsymbol{T}_{2}$ on the other. The distributions of the cation concentrations $\mathrm{c}$, and $\mathrm{c}$, with the charge number $\boldsymbol{z}_{1}$ and $\mathrm{z}_{2}$ respectively, and anion concentration c, with the charge number $z_{3}$ are determined by the system of NernstPlank equations:

$$
\begin{gathered}
j_{1}=z_{1} F\left(D_{1} \frac{\mathrm{d} c_{1}}{\mathrm{~d} x}+u_{1} c_{1} \frac{\mathrm{d} \phi}{\mathrm{d} x}+D_{1}^{\mathrm{T}} \frac{\mathrm{d} T}{\mathrm{~d} x}\right), \\
j_{2}=z_{2} F\left(D_{2} \frac{\mathrm{d} c_{2}}{\mathrm{~d} x}+u_{2} c_{2} \frac{\mathrm{d} \phi}{\mathrm{d} x}+D_{2}^{\mathrm{T}} \frac{\mathrm{d} T}{\mathrm{~d} x}\right), \\
0=D_{3} \frac{\mathrm{d} c_{3}}{\mathrm{~d} x}-u_{3} c_{3} \frac{\mathrm{d} \phi}{\mathrm{d} x}+D_{3}^{\mathrm{T}} \frac{\mathrm{d} T}{\mathrm{~d} x}, \\
z_{1} c_{1}+z_{2} c_{2}=z_{3} c_{3} .
\end{gathered}
$$

Here $D_{k}, u_{k}$ and $D_{k}^{\top}$ are diffusion coefficients, mobility and thermal diffusion coefficient of the kth ion $(\mathrm{k}=1,2,3)$, respectively, $\mathbf{j}_{\mathbf{1}}$ and $\boldsymbol{j}_{\mathbf{2}}$ are current densities, transported by ion species 1 and $2, F$ is the Faraday number, $\boldsymbol{\phi}$ is the electric potential and $\mathrm{x}$ is the coordinate, directed from the left electrode $(\mathrm{x}=$ $\mathrm{x}$,$) towards the right one \left(\mathrm{x}=\boldsymbol{x}_{\mathbf{R}}\right), \boldsymbol{x}_{\mathbf{L}} \mathbf{I} \mathrm{x} \leq \boldsymbol{x}_{\mathbf{R}}$. Relation (13) expresses the condition of electroneutrality.

It follows from the stoichiometry of the electrochemical reaction, that

$$
\frac{j_{1}}{z_{1}}=-\frac{j_{2}}{z_{2}}
$$

and, consequently, the density of the integral electric current density can be presented as

$$
j=j_{1}+j_{2}=j_{1}\left(1-z_{2} / z_{1}\right) .
$$

The cathode reaction on one electrode occurs in stationary conditions with the same rate, as the anode reaction on the other electrode. That is why the total amount of substance of each sort remains constant and is defined by the initial concentrations only:

$$
\int_{x_{L}}^{x_{k}} c_{k}(x) \mathrm{d} x=\left(x_{\mathrm{R}}-x_{\mathrm{L}}\right) c_{k}^{0} .
$$


Beside the two boundary conditions are valid on each electrode:

$$
\begin{aligned}
j= & j_{0}\left(\frac{\left.c_{1}\right|_{x_{\mathrm{L}}}}{c_{1}^{0}} \exp \left(\frac{-\left(z_{1}-z_{2}\right)(1-\alpha) F \xi_{\mathrm{L}}}{R T_{1}}\right)\right. \\
& \left.-\frac{\left.c_{2}\right|_{x_{\mathrm{L}}}}{c_{2}^{0}} \exp \left(\frac{\left(z_{1}-z_{2}\right) F \alpha \xi_{\mathrm{L}} \mathrm{J}}{R T_{1}}\right)\right), \\
j= & j_{0}\left(-\frac{\left.c_{1}\right|_{x_{\mathrm{R}}}}{c_{1}^{0}} \exp \left(\frac{-\left(z_{1}-z_{2}\right)(1-\alpha) F \xi_{\mathrm{R}}}{R T_{2}}\right)\right. \\
& \left.+\frac{\left.c_{2}\right|_{x_{\mathrm{R}}}}{c_{2}^{0}} \exp \left(\frac{\left(z_{1}-z_{2}\right) F \alpha \xi_{\mathrm{R}}}{R T_{2}}\right)\right) .
\end{aligned}
$$

For simplicity, we will further assume $\alpha=\frac{1}{2}$. The potential drop $u$ between two electrodes is the algebraic sum of the potential drops on the electrolyte solution boundaries and inside the electrolyte.

$$
\begin{aligned}
u=-\left(\left.\phi^{(s)}\right|_{T=T_{2}}+\xi_{\mathrm{R}}\right)+\left(\left.\phi\right|_{x=x_{\mathrm{R}}}-\left.\phi\right|_{x=x_{\mathrm{L}}}\right) \\
+\left(\left.\phi^{(\mathrm{s})}\right|_{T=T_{1}}+\xi_{\mathrm{L}}\right) .
\end{aligned}
$$

We assume, that the Einstein correlation for mobility is valid:

$$
u_{\mathbf{k}}=D_{\mathbf{k}} \frac{F z_{\mathbf{k}}}{R T}
$$

that is, strictly speaking, true for diluted solutions only. Obviously, the mobilities and the diffusion coefficients also depend on the temperature. But we suggest, that the temperature difference is not too high, so that the mobilities and the diffusion coefficients can be considered constant in the whole volume.

It can be verified a posteriori, that the arising concentration difference is insignificant, therefore the mobilities and diffusion coefficients dependence upon the concentrations can be definitely neglected.

Expressions (10)-(20) are complex system of differential, algebraic and integral (16) equations. In order to find the current-voltage dependence $U(I)$ from (10)-(20), it is necessary that the profiles of concentrations and potential should be determined. Differential equations (10)-(12)are nonlinear, because they include products of two unknown functions: concentration and potential. Boundary conditions are also nonlinear (with the exponential terms).

Here we are going to present two simplified solutions: one neglecting the migration transport and another neglecting the thermal diffusion transport. These results will be afterwards compared.

In the absence of the convection flow, the term corresponding to the thermal diffusion, is simply $\mathbf{d} \mathbf{T} / \mathrm{d} \boldsymbol{x}=\left(\mathrm{T}_{2}-T_{1}\right) /\left(\boldsymbol{x}_{\mathbf{R}}-\boldsymbol{x}_{\mathbf{L}}\right)$. As far as the migration terms in (10)-(13) are negligible the expression for the concentration profiles can be found immediately:

$$
c_{\mathrm{k}}=c_{\mathrm{k}}^{0}+\left(x-\frac{x_{\mathrm{L}}+x_{\mathrm{R}}}{2}\right) \frac{i_{\mathrm{k}} /\left(z_{\mathrm{k}} F\right)-D_{\mathrm{k}}^{\mathrm{T}} \mathrm{d} T / \mathrm{d} x}{D_{\mathrm{k}}}
$$

Substituting this expression into (17) and (18), we obtain simple equations for $\xi_{L}$ and $\xi_{R}$ which can be solved as square equations. The resulting currentvoltage curve is shown in Fig. 3 (Curve b).

The other case, when migration occurs, requires much more cumbersome considerations. Anyway,

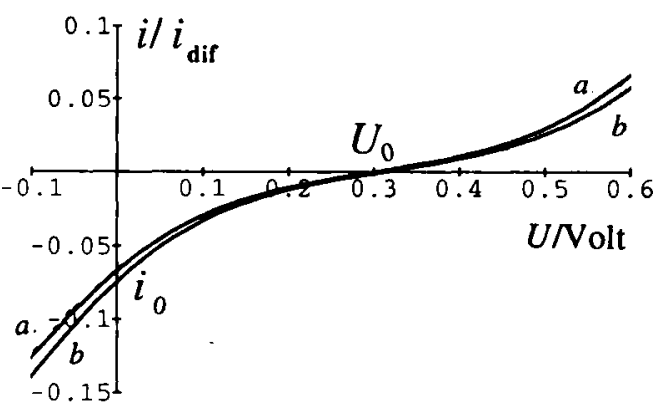

Fig. 3. The current-voltage curve for systems $\left[\mathrm{Fe}(\mathrm{CN})_{6}\right]^{3-} \mid\left[\mathrm{Fe}(\mathrm{CN})_{6}\right]^{4-}$ in the case of stagnant electrolyte: (a) diffusion-migration transport; (b) diffusion and thermodiffusion. $\quad c_{0}^{1}=c_{2}^{0}=1 \mathrm{M}, \quad \mathrm{D},=\mathrm{D},=4$ $\times 10^{-3} \mathrm{~cm}^{2} \mathrm{~s}^{-1}, \mathrm{~L}=1 \mathrm{~mm}, j_{0}=100 \mathrm{Am}^{-2}, D_{1}^{\mathrm{T}}=D_{2}^{\mathrm{T}}=\mathrm{D}$, $x 10^{-3} \mathrm{MK}^{-1}, \quad T=30 \mathrm{~K}$. The limiting diffusion current density for anions is scale for current.

this complex mathematical problem allows one to get an exact analytical solution, that does not require any additional approximations or linearization. This solution in case of electrodes with equal temperature and the only electroactive species of ions was given in [14]. The principal steps of generalisation of that solution for the case of different electrode temperatures and two electroactive ions, and also the final formulas for the current-voltage dependency, are given in Appendix A.

The main principle of the solution [14] is that instead of seeking the direct dependence $j(U)$, the parametrical dependencies $\boldsymbol{j}(\omega)$ and $\boldsymbol{U}(\omega)$ should be determined. The dimensionless qrop of potential in the tran zone $\left.\boldsymbol{\phi}\right|_{x=x_{k}}-\left.\phi\right|_{x=x_{1}}$ is used as an intermediate parameter $\omega$. With these expressions for $j(\omega)$ and $U(\omega)$ it is possible to plot the curve for the current-voltage dependence, as well as by means of functions $j(U)$ or $U(\mathrm{j})$.

The current-voltage curve obtained by theoretical calculations for one of systems $\left[\mathrm{Fe}(\mathrm{CN})_{6}\right]^{3-} \mid\left[\mathrm{Fe}(\mathrm{CN})_{6}\right]^{4-}$, studied experimentally in detail in a number of papers [7-9], is given in Fig. 3 (Curve a). Thus, the expressions (17)-(19) give the elementary expressions of the current-voltage dependence $j(\mathrm{U})$.

It is shown in Fig. 3 that the both curves begin at the same point $\boldsymbol{U}_{\mathrm{o}}$ on the axis $\mathrm{j}$, related to the open circuit. In point $U_{0} / 2$, corresponding to the optimum regime of TGC functioning, the curves can be considered a not distinguishable. The noticeable divergencies appear near the region of the short circuit and further towards high values of voltage that are not realised in practice.

One can note that the current-voltage curve deviates from a straight line. The simple explanation of this is the following. The electrochemical reaction (5) is supposed to be reversible. Reversible electrochemical reactions have only one inflection point on the $\mathrm{V}-\mathrm{A}$ curve near zero currents. The shape of the curve on the Fig. 3 is different and shows that the reaction under investigation is, really, irreversable. The typical value of the exchange current density for such a system is about $100 \mathbf{A ~ m}^{-2}$ which is less than the typical value of the diffusion limiting current density. For detail discussion see[9, 12]. 
Another advantage of the exact solution given in the Appendix A is worth mentioning. For the fixed value of $\omega$, ie for the certain current density $\mathbf{j}$ and the potential difference $U$, it is possible to plot the profiles of concentrations $\boldsymbol{c}_{\mathbf{i}}(\boldsymbol{x})$ (see Fig. 4). As well as the curve $\boldsymbol{j}(\boldsymbol{U})$, these curves are plotted in parametrical form of $\left(c_{i}(\phi), x(\phi)\right)$; the value of the electric potential $\phi$ in the transport zone volume is used as an intermediate parameter. These profiles of concentrations are given for the above mentioned electrochemical system in the regime of the maximum normalised efficiency, ie at $U=U_{0} / 2$. In the same figure, the integral concentration $\mathrm{c}=\boldsymbol{c}_{\mathbf{1}}+\boldsymbol{c}_{\mathbf{2}}+\boldsymbol{c}_{\mathbf{3}}$ is given. One can see that although the concentrations $c_{1}$ and $c_{2}$ vary, their changes compensate each other, so that the difference of the total concentration Ac and concentration of anions $\Delta c_{3}$ is not significant: $\Delta \mathrm{c} \ll c$. It also proves our suggestion, that the coefficients of diffusion and mobility can be considered approximately independent of the concentrations (because concentrations themselves do not change significantly).

\section{Convective flow of the liquid in TGC}

Let us consider an ordinary TGC with vertical electrodes, where the distance between the electrodes is much less than the cell size (Fig. 5). In such a cell the two dimensional liquid flow with the velocity $v$, which can be described by the stationary NavierStokes equation with additional terms in Bousinesque form, arises:

$$
-\frac{1}{\rho} \nabla p+v \Delta \vec{b}-\beta g\left(T-T_{0}\right) \vec{e}_{y}+\bar{\beta}_{g}\left(c-c_{0}\right) \vec{e}_{y}=0 .
$$

Here $\mathrm{p}$ is pressure, $\boldsymbol{v}$ is kinetic viscosity, $\mathrm{g}$ is the gravitation -constant, $\beta$ is the thermal expansion coefficient, $\beta$ is the coefficient of dependence of the density on concentration, $\vec{e}_{y}$ is the ort in the direction of axis y, $\boldsymbol{T}_{\mathbf{0}}, \boldsymbol{c}_{\mathbf{0}}$ and $\rho$ are the average values of the temperature, concentration and density, respectively. The numerical solution of such a double diffusion problem is very complicated [15]. The characteristic rate of the liquid motion $|\boldsymbol{v}|$ is extremely low: $|v| \sim 10^{-4} \mathrm{~m} \mathrm{~s}^{-1}$, the characteristic size of the system (the distance between the electrodes) is

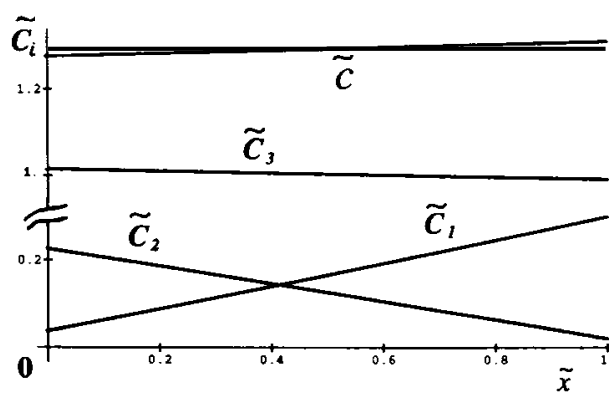

Fig. 4. The dimensionless concentration profiles of ions, corresponding to the optimum regime. Other parameters as for Fig. 3. Note, that the distance between electrodes is used as the length scale, the average concentration of anions is scale for concentration.

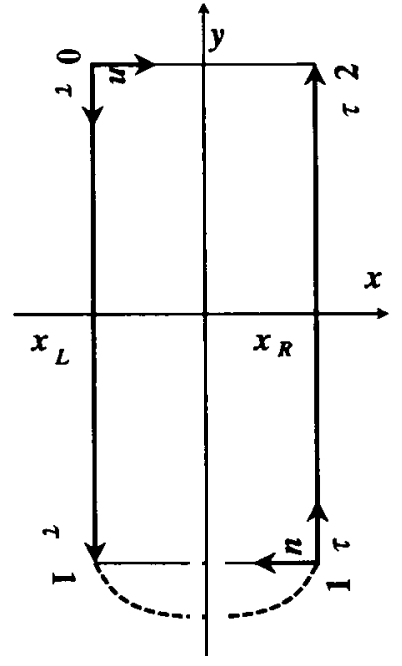

Fig. 5. The scheme of a TGC for calculation of diffusionconvection transport.

$\sim 1 \mathbf{0}^{-3} \mathrm{~m}, \boldsymbol{v}$ for water is approximately equal to $10^{-6} \mathrm{~m} \mathrm{~s}^{-1}$. Therefore, the Reynolds number is $\operatorname{Re}-0.1$, that gives the ground to neglect the nonlinear term in (21).

The pressure gradient term can be eliminated by taking the curl of equation (21). In terms of the stream function, which is correlated to $v$ by the expression $\overrightarrow{\boldsymbol{v}}=(\partial \Phi / \partial \boldsymbol{y},-\partial \boldsymbol{\Phi} / \partial \boldsymbol{x})$, we determine that

$$
{ } \Delta^{2} \Phi=\beta g \frac{\partial T}{\partial x}-\bar{\beta} g \frac{\partial c}{\partial x}
$$

We can significantly simplify the consideration if we compare the typical values of two terms in the right part of (22). In accordance with the results of reference [9], if the convective flow of liquid takes place under the influence of the temperature difference of $50 \mathrm{~K}$, the arising difference of concentrations is $0.08 \mathrm{M}$. Introducing $\beta=3 \times 10^{-4} \mathrm{~m}^{3} \mathrm{~K}^{-1}$ and $\overline{\boldsymbol{\beta}}=5 \times 10^{-2} \mathrm{~m}^{3} \mathrm{~mol}^{-1}$ in accordance with the table data from [16, 17], we get that the first term in the right part of (22) is four times as much as the second term. It means that the convection flow of liquid takes place mainly due to the temperature gradient, but not due to the concentration gradient.

The other simplification is connected with the fact that in the main bulk volume of liquid the thermal flow density from one electrode to another is constant, that provides for the temperature profile remaining linear [9]. Some deviations from linearity are observed in the upper and lower parts of the cell, where the convective thermal transport becomes significant. It occurs because there is the heat transport from one electrode to another in the main volume of liquid, ie in the horizontal direction, and the liquid flow is directed upwards or downwards, ie in the perpendicular direction and cannot cause any significant convective heat transport. These reasons were also supported by computer modelling; its detailed description is beyond the scope of this paper.

Thus, in the middle part of the cell (excluding two approximately square upper and lower domains) the derivative $\partial T / \partial x$ can be replaced by the constant 
temperature gradient and we can present (22) in the following form:

$$
\Delta^{2} \Phi=\Theta
$$

where

$$
\Theta=\frac{\beta g\left(T_{2}-T_{1}\right)}{v\left(x_{\mathrm{R}}-x_{\mathrm{L}}\right)}
$$

is a certain constant. The equation of the viscous liquid motion (23) is to be completed by boundary conditions in the absence of tangential rate component on the electrode surface:

$$
\left.\frac{\partial \Phi}{\partial x}\right|_{x=x_{L}}=0 ;\left.\frac{\partial \Phi}{\partial x}\right|_{x=x_{m}}=0
$$

and also by the condition of the absence of the normal rate component. The latter is equivalent to the statement that the boundary between the liquid and solid phases is one of $\Phi=$ const. and the constant can be chosen arbitrarily:

$$
\left.\Phi\right|_{x=x_{R}}=0 ;\left.\Phi\right|_{x=x_{L}}=0 .
$$

In the main volume the liquid flow does not depend on boundary conditions on the upper and lower boundaries of the cell. Equation (23), with boundary conditions (25) and (26), gives the elementary solution:

$$
\Phi=0.25 \Theta\left(x-x_{\mathrm{L}}\right)^{2}\left(x-x_{\mathrm{R}}\right)^{2}
$$

or, for the rate:

$$
\vec{v}=\Theta\left(x-x_{\mathrm{L}}\right)\left(x-x_{\mathrm{R}}\right)\left(x-\frac{x_{\mathrm{L}}+x_{\mathrm{R}}}{2}\right) \vec{e}_{\mathrm{y}} .
$$

The liquid is stagnant along the vertical middle line due to the axial symmetry of TGC. Expression (28) will be used further for solving the problem of diffusion-convective flows of cations under the influence of the forced convection. So, the general problem of diffusion-convective transport of mass and energy can be divided into three consequent steps: (1) calculating of the heat transport problem; (2) solving of the subproblem of the generation of natural convection of liquid; (3) solving of the subproblem of cations of two species transport under the influence of the diffusion and the given fixed convection.

Such a division is mainly stimulated by the physical sense of the problem. The existence of the temperature difference between the electrodes (the first-order effect) leads to two consequences (the second-order effects): appearance of the heat convection in liquid and of the electric current flow. The latter causes a certain redistribution of concentrations, and it leads to the concentrational convection of liquid (the fourth-order effect).

Certainly, the quantitative evaluations are necessary in order to support these qualitative considerations, and that was done at the beginning of this part, but these concentrations provide for better understanding of the reason why the heat convection should be taken into account (the second-order effect) and the concentrational convection should not (the fourth-order effect).
6. Diffusion-convection currents in TGC with vertical electrode arrangement

The diffusion coefficients of ions are much less than the heat conductivity coefficient and, consequently, the processes of ion transport and energy transport in TGC occur in two absolutely different way (see part 5).

In general, the cation transport from one electrode to another can be divided into three stages:

(1) Diffusion transport of an ion from the electrode surface inside the moving liquid.

(2) Convection transport along the surface to its end; then, in the upper (or lower) part of the cell ions are transferred from one electrode to another by the liquid flow; then they keep moving along the surface of the other electrode.

(3) Diffusion transport from the moving liquid towards the other electrode.

So, all the changes of concentration are located in the thin boundary layer near the electrodes and the initial values of all components remain constant in the main central volume.

We take into account the fact that the cell height $h$ is much more than its width $\mathrm{L}=(\mathrm{x},-\mathrm{x}$,$) , and$ introduce the dimensionless "surface" system of coordinate $(n, \tau)$. The origin of this new coordinate system is the upper left corner of the cell, the normal axis $\vec{n}$ is directed from the electrode into the solution, and the tangential axis $t$ consists of two components: the first one is directed along the surface of the first electrode downwards, and the second one is directed along the surface of the second electrode upwards (see Fig. 5); both components are directed along the direction of the liquid flow. There is a segment of the length $\mathrm{L}$ that is missing at the joint point of those two components. But there are no electrode processes in this region, and its length is much less than the length of the electrodes $\mathrm{L} \ll \mathrm{h}$; so we can expect that the concentration and its derivative will be practically continuous in this point. The same considerations can be applied to the other joint point in the origin. The dimensionless equation of the convective diffusion [13] for cations of the kind $\mathrm{m}$ is:

$$
D_{m} \frac{\partial^{2} c_{m}}{\partial n^{2}}=\frac{\partial c_{m}}{\partial \tau} n\left(\frac{\Theta L^{2} h^{2}}{2}\right), \quad m=1,2
$$

where the value of $h$ is chosen as the length scale. In equation (29) the common approximation for transport in the boundary layer was done: in the expansion of the rate function $\mathrm{V}$ along the coordinate $\mathrm{x}$ only the linear term was left at $\mathrm{x}=\boldsymbol{x}_{\mathbf{R}}$ ( or $\mathrm{x}=\mathrm{x}$,), that relates to the absence of the lateral diffusion, as the convective transport is much more significant in that direction.

Functions $\mathrm{c}$, and $\boldsymbol{c}_{2}$ are to be continuous in the points $\mathrm{r}=1$ and $\mathrm{r}=2(\tau=0)$, ie to be periodical functions of the coordinate $\boldsymbol{\tau}$ with the period 2 . Besides the solution of equation (29) tend to the volume values $c_{1}^{0}$ and $c_{2}^{0}$ at a long distance from the electrode, ie when $\boldsymbol{n}$ has a rather high value.

Although equations (29) look rather simple, they allow us to describe well all three stages of transport, listed at the beginning of this chapter. The main difficulty in their solving is that the boundary conditons 
inevitably include both surface concentrations $\left.c_{\mathbf{1}}\right|_{\mathbf{s}}$ and $\mathbf{c}_{2} \mid \mathbf{s}$. That is why both equations (29) are to be solved simultaneously.

Migration transport is not accounted for in the problem (29), which means that the supporting electrolyte is in excess, or that the electric potential in the transport zone can be considered constant. Thus, the voltage drop (and the overvoltage) between the electrode and the bulk solution can be assumed constant along the electrode surface. It makes the boundary conditions (6) linear relative to concentrations $c_{1}$ and $c_{2}$, and it allows us to apply the common methods of mathematical physics. Such a solution, obtained by means of Fouriertransformation and expansion by Airy functions, is given in the Appendix B.

A few examples of the concentration profiles $c_{1}$ $-c_{1}^{0}$, in the horizontal sections of the cell are presented in Fig. 6. Liquid motion in the cell moves counter clockwise. It can be shown that as an elementary volume, taken beside the left electrode, moves down, it is being gradually saturated by the cations 1 , and as it moves upwards along the other electrode, it loses its cation supply and gets to the upper part of the cell short of cations (see Fig. 6). The curve for $\boldsymbol{c}_{\boldsymbol{2}}-\boldsymbol{c}_{\mathbf{2}}^{\mathbf{0}}$ differs from Figs 6 and 7 only in the negative factor, that means that the shortage of cations of kind 2 appears not in the upper, but in the lower part of the cell. Three-dimensional surface, corresponding to the concentration distribution in the volume of TGC, is shown on the Fig. 8.

The current-voltage curve for the case of diffusion-convection transport looks similar to the ones for the diffusion-migration case, but characteristic values are different. Let us estimate the maximal possible decreasing of the resistance due to the convection flow. Suppose that convection transfers ions from one cell to another fast and that resistance occurs mostly due to slow diffusion transport in boundary layers. Of course, this approximation gives us over-estimated values of efficiency. The thickness of the diffusion layer on one electrode is about the scale of changing first term in the series for concentration profile (see equation (B4)). Therefore, we can estimate the part of the cell occupied by two diffusion layers as

$$
2 \sqrt[3]{\frac{\pi \Theta^{2} H^{2}}{2 D_{1} L}}
$$

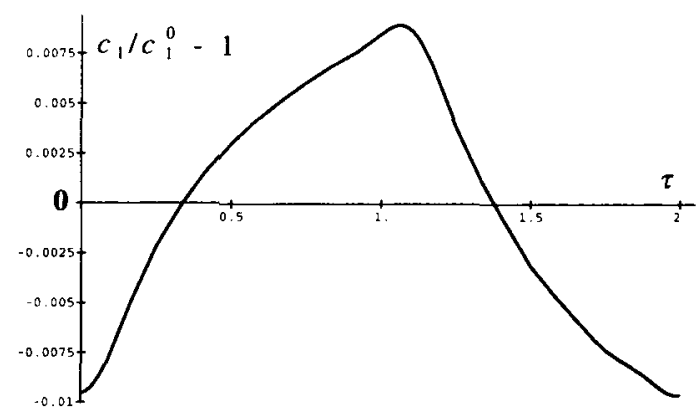

Fig. 6. The deviation of concentration of cations of the first kind along surfaces of the first electrode $(0<\tau<1)$ and of the second one $(1<\tau<2)$.

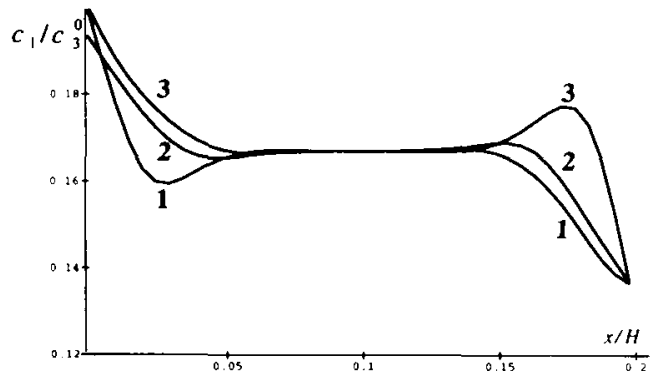

Fig. 7. The dimensionless concentration profiles of cations of the first kind in the horizontal sections of TGC: (1) upper part; (2) medium part; (3) lower part.

In most cases the value of (30) is around $\frac{1}{3}$. If it is less than 1 , the diffusion layers overlap and, actually, there is no convection transport. For high values of 8 , ie temperature difference, the convection transport of heat becomes important, so that normalised efficiency reaches its limiting value. In general, we approved the conclusion of [7-10] that the convection transport can increase the efficiency of TGC up to 2-3 times.

\section{CONCLUSION}

In the present work the functioning of existing thermogalvanic cells has been theoretically studied. The analysis for the simplest case with account for only diffusion transport of the reacting substances from one electrode to another, as well as for more complex cases, has been made: diffusion-migration transport for the horizontally arranged cell and diffusion-convection transport for the vertically arranged one. In all cases the current-voltage curves were calculated for TGC.

The qualitative analysis of the efficiency of TGC functioning has been performed. The brief algorithm for choosing the optimum parameters is the following:

(1) The solvent with the lowest thermal conductivity should be chosen. Water solution cannot be considered as a good one, although it is generally used.

(2) The mixture of two substances, which can transform one into another by the reversible electrochemical reaction like (5), should be chosen. The maximum changes of the equilibrium potential (5) with temperature should be possibly reached. The reaction (5) must be the reversible one, ie the exchange current density must be higher than the limiting diffusion current density (the constant resistance of the cell).

(3) The temperature range for the working electrodes should be chosen, to provide for the optimum potential changes (see the previous item).

(4) The concentrations of the components are to be high enough, but it should be taken into account that the growth of the efficiency with the concentration is limited. Moreover, in the case of the horizontally arranged cell, when the migration transport is possible, too high concentration under certain conditions can lead to the following: one substance 


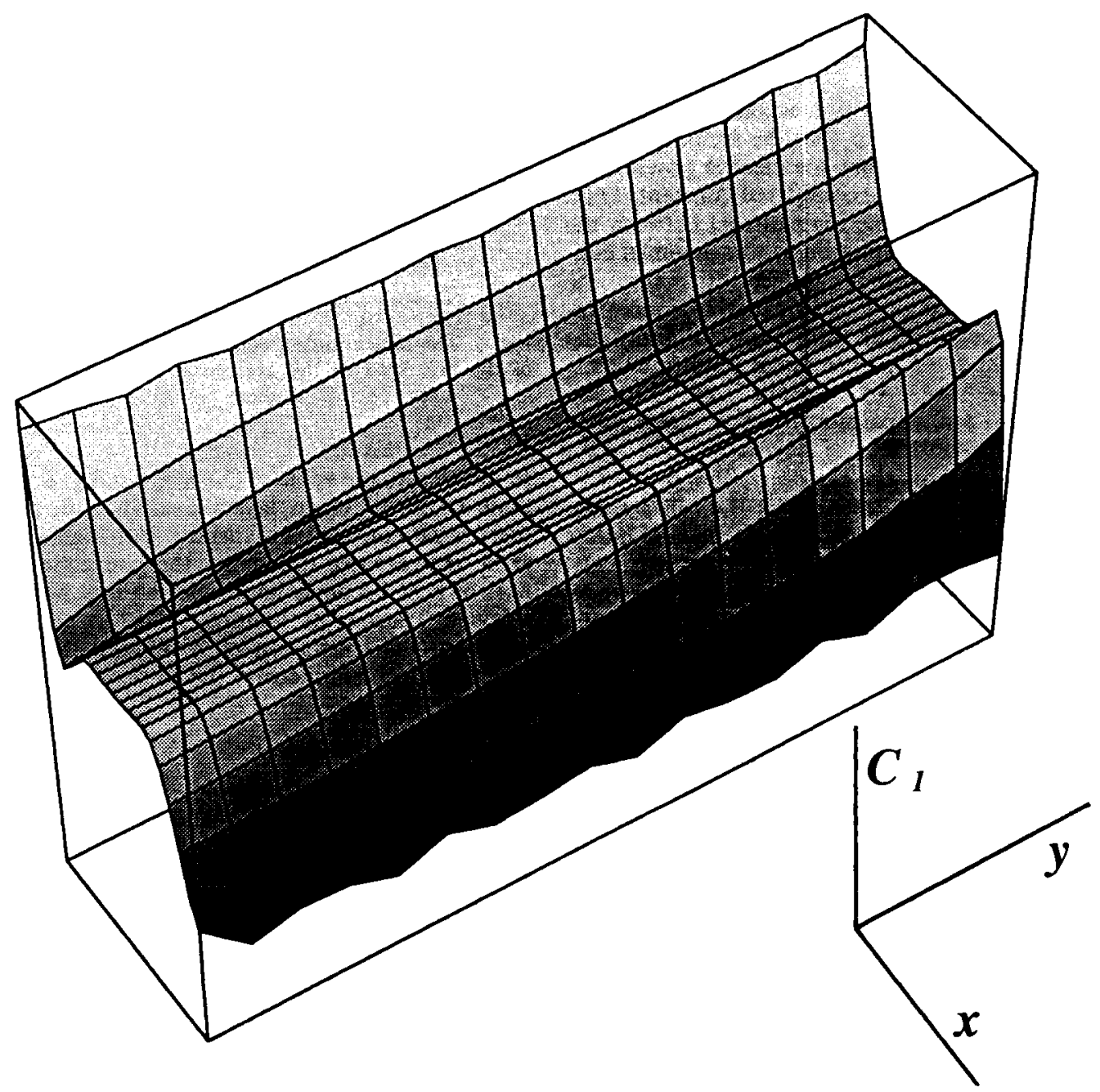

Fig. 8. The concentration distribution of cations of the first kind as a function of coordinates $\boldsymbol{x}$ and $\boldsymbol{y}$ (see also Figs 6 and 7).

becomes a supporting electrolyte for another one, which can lead to reduction of the rate of matter transport and reduction of $\eta$.

(5) The external resistance should be permanent (switches on and off are impossible) and have the same value as TGC itself.

(6) Geometrical sizes of electrodes, the distance between them and their arrangement (horizontal or vertical), the temperature difference between the electrodes and the rate of liquid circulation inside the cell have a minor influence on the results compare with points (1)-(5) (see discussion in the end of section 6).

It is still a question of discussion if the researchers succeed to find the optimum parameters of TGC by means of this algorithm. Now we have to admit, that the theoretical analysis of existing cells gives approximately the same evaluation for their efficiency, $\eta \sim 0.1 \%$, as experimental measurements. Undoubtedly, it is not enough for any practical applications.

We think, that the further progress in this field will be connected with TGC of principally new type [18,
19]. in which the main volume between the electrodes is filled by gas phase.

Acknowledgements - This work was supported by Swedish Academy of Science. The author is grateful to the referee, Prof. F. Bark, Prof. Yu. I. Kharkats and Dr T. Ikeshoji for useful discussions.

\section{REFERENCES}

1. B. Burrows, Proc. Inters. Energy Convers. Eng. Con/. 10, 821 (1975).

2. B. Burrows. J. Electrochem. Soc. 123, 154 (1976).

3. H. L. Chum, Proc. Inters. Energy Convers. Eng. Conf. 15, $1603(1980)$.

4. T. 1. Quickenden and C. F. Vernon, Sol. Energy 36, 63 (1986)

5. J. N. Agar, in Advances in Electrochemistry and Electrochem. Eng. (Edited by P. Delay) Vol. 3, p. 31. Interscience, New York (1963).

6. R. Haase, Thermodynamics of Irreversible Processes, Dover Publications, New York (1990).

7. T. Ikeshoki, Bull.Chem. Soc. Jpn, 60, 1505(1987).

8. T. Ikeshoji, J.electroanal. Chem. 296, 19 (1990). 
9. T. Ikeshoji and F. N. Nahui, J. electroanal. Chem. 305, 147 (1991).

10. T. Ikeshoji, F. N. Nahui, S. Kimura and M. Yoneya, J. electroanal. Chem. 312,43 (1992).

11. V. Bagozkii, Background of Electrochemistry, Chimija, Moscow (1988)(in Russian).

12. K. Vetter, Electrochimsche Kinetik. Springer-Verlag, Berlin (1961).

13. V. G. Levich, Physicochemical Hydrodynamics, Prentice-Hall, Englewood Cliffs, New Jersey (1962).

14. A. V. Sokirko and F. Bark, 44th Meet. of Int. Soc. of Electrochemistry, p. 626. Berlin, Germany (1993).

15. F. Alavyoon. Proc. Inter. Sem. Heat and Mass Transfer in Porous Media, Hemisphere Publishing (1991).

16. J. Newman, Electrochemical Systems. 2nd ed. PrenticeHall, Englewood Cliffs, New Jersey (1991).

17. G. K. Batchelor, An Introduction to Fluid Dynamics. Cambridge (1988).

18. T. Ikeshoji and F. N. Nahui, 43rd Meet. of Int. Soc. of Electrochemistry. Cordoba, Argentina p. 299 (1992).

19. Y. Maeda, E. Itoh and M. Inagaki, Synth. Metals 20.73 (1987).

20. Handbook of Mathematical Functions, (Edited by M. Abramowitz and I.-N. Y. Stegun, National Bureau of Standards, Gaithersburg, Maryland (1964).

\section{APPENDIX A}

In this appendix the solution of the equation system (10)-(20) is presented and the parametric expression for the current-voltage dependence of TGC is obtained.

Let us introduce the dimensionless variables:

$$
\begin{aligned}
& \mathrm{Z}_{1}=z_{1} / z_{3} ; \quad \mathrm{Z}_{2}=z_{2} / z_{3} ; \quad \tilde{c}_{m}=c_{m} / c_{3}^{0} ; \\
& \tilde{x}=\frac{x-x_{L}}{x_{R}-x_{1}} \\
& K=\frac{z_{1} c_{1}^{0}}{z_{3} c_{3}^{0}} ; \quad \psi_{1}=\psi(\tilde{x}=0) ; \quad \psi_{2}=\psi(\tilde{x}=1) \\
& \tilde{j}=\frac{j\left(x_{\mathrm{R}}-x_{1}\right)}{F D_{1} c_{3}^{0}} ; \quad \chi=\frac{F D_{1} c_{3}^{0}}{\left(x_{\mathrm{R}}-x_{\mathrm{L}}\right) j_{0}} \frac{z_{1}-z_{2}}{z_{1}-z_{2} D_{1} / D_{2}} ; \\
& w_{1}=\exp \left(\frac{\left(z_{1}-z_{2}\right) \xi_{L} F}{2 R T_{1}}\right) ; \quad w_{2}=\exp \left(\frac{\left(z_{1}-z_{2}\right) \xi_{R} F}{2 R T_{2}}\right) \\
& \psi=z_{3} \frac{F}{R T} \phi
\end{aligned}
$$

Then the Nernst-Planck equations, condition of electroneutrality and boundary conditions may be presented as follows:

$$
\begin{gathered}
\frac{\tilde{j}}{z_{1}-z_{2}}=\frac{\mathrm{d} \tilde{c}_{1}}{\mathrm{~d} \tilde{x}}+Z_{1} \tilde{c}_{1} \frac{\mathrm{d} \psi}{\mathrm{d} \tilde{x}} ; \\
-\frac{\tilde{j}}{z_{1}-z_{2}} \frac{D_{1}}{D_{2}}=\frac{\mathrm{d} \tilde{c}_{2}}{\mathrm{~d} \tilde{x}}+Z_{2} \tilde{c}_{2} \frac{\mathrm{d} \psi}{\mathrm{d} \tilde{x}} ; \\
0=-\frac{\mathrm{d} \tilde{c}_{3}}{\mathrm{~d} \tilde{x}}+\tilde{c} \frac{\mathrm{d} \psi}{\mathrm{d} \tilde{x}}, \\
Z_{1} \tilde{c}_{1}+Z_{2} \tilde{c}_{2}=\tilde{c}_{3} \\
\int_{0}^{1} \tilde{c}_{3} \mathrm{~d} \tilde{x}=1 ; \int_{0}^{1} \tilde{c}_{2} \mathrm{~d} \tilde{x}=(1-K) / Z_{2}, \\
\chi J=\frac{\tilde{c}_{1}(\tilde{x}=0) Z_{1}}{K w_{1}}-\frac{\tilde{c}_{2}(\tilde{x}=0) Z_{2} w_{1}}{1-K} ;
\end{gathered}
$$

where

$$
\begin{gathered}
-\chi J=\frac{\tilde{c}_{1}(\tilde{x}=1) Z_{1}}{K w_{2}}-\frac{\tilde{c}_{2}(\tilde{x}=1) Z_{2} w_{2}}{1-K} ; \\
J=\frac{Z_{1}-Z_{2} D_{1} / D_{2}}{Z_{1}-Z_{2}} \tilde{j}
\end{gathered}
$$

Multiplying (A2) by Z, (A3) by Z, and summing them up together with (A4), weget:

$$
\frac{\mathrm{d} \tilde{x}}{\mathrm{~d} \psi}=\left(\frac{\mathrm{d} \psi}{\mathrm{d} \tilde{x}}\right)^{-1}=\left(Z_{2}\left(Z_{2}-Z_{1}\right) \tilde{c}_{2}+\left(Z_{1}+1\right) \tilde{c}_{3}\right) / J
$$

(A10)

Expression (A10) allows us to transfer the independent variable $\tilde{x}$ in equations (A2-(A4) for a new independent variable $\psi$. It immediately results from (A4), that $\tilde{\boldsymbol{c}}_{\mathbf{3}} \sim \exp \psi$, and it results from (A3), that $\tilde{\boldsymbol{c}}_{\mathbf{2}}$ is the linear combination of exponential functions of potential $\exp \psi$ and $\exp \Lambda \psi$, where A is:

$$
\Lambda=\frac{D_{1}-D_{2}}{D_{2} / z_{2}-D_{1} / z_{1}}
$$

The unknown coefficients in the exponents are to be determined from the boundary conditions. It is convenient to represent the solution for $\tilde{\boldsymbol{c}}_{\mathbf{2}}, \tilde{\boldsymbol{c}}_{\mathbf{3}}$ in the form

$$
\begin{gathered}
\tilde{c}_{3}=M \mathrm{e}^{\psi-\psi_{1}} \\
\tilde{c}_{2}=M \mathrm{e}^{\psi-\psi_{1}}\left(s+N \mathrm{e}^{\left(\Lambda-1 M \psi-\psi_{1}\right)}\right)
\end{gathered}
$$

where the constant $s$ is calculated directly from the transformed equation (A3):

$$
s=\frac{\left(Z_{1}+1\right) D_{1}}{\left(Z_{1} D_{2}-Z_{2} D_{1}\right)(\Lambda-1)}
$$

In order to determine constants $\mathrm{M}$ and $\mathrm{N}$, the integration variable in (A6) is changed and then (A10) is integrated, after that the obtained square equation should be solved relative to $\mathrm{N}$ :

$$
\begin{aligned}
& N=N(\omega) \\
& =\left[\left(\gamma_{2}-\frac{1-K}{Z_{2}} \gamma_{4}\right)^{2}\left(\omega^{\Lambda+1}-1\right)^{2}-4 \gamma_{1}\left(\omega^{2 \Lambda}-1\right)\right. \\
& \left.\quad \times\left(\gamma_{3}-\frac{1-K}{Z_{2}} \gamma_{5}\right)\left(\omega^{2}-1\right)\right]^{1 / 2}-\left[\left(\gamma_{2}-\frac{1-\bar{K}}{Z_{2}} \gamma_{4}\right)\right. \\
& \left.\times\left(\omega^{\wedge+1}-1\right) \frac{1}{2 \gamma_{1}\left(\omega^{2 \Delta}-1\right)}\right], \\
& M=M(\omega)=\frac{N\left(\omega^{\wedge}-1\right) \gamma_{6}+(\omega-1) \gamma_{7}}{N\left(\omega^{\wedge+1}-1\right) \gamma_{4}+\left(\omega^{2}-1\right) \gamma_{5}} \\
& J=J(\omega)=M\left[N\left(\omega^{\wedge}-1\right) \gamma_{6}+(\omega-1) \gamma_{7}\right] .
\end{aligned}
$$

(A14)

The designations are introduced for the potential drop in the transport region $\log \omega=\boldsymbol{\psi}_{2}-\boldsymbol{\psi}_{\mathbf{1}}$ and for cumbersome algebraic combinations of charge numbers and diffusion coefficients:

$$
\begin{aligned}
& \gamma_{1}=Z_{2}\left(Z_{2}-Z_{1}\right) / 2 \Lambda \\
& \gamma_{2}=\left[2 Z_{2}\left(Z_{2}-Z_{1}\right) s+Z_{1}+1\right] /(\Lambda+1) \\
& \gamma_{3}=s\left[Z_{2}\left(Z_{2}-Z_{1}\right) s+Z_{1}+1\right] / 2 \\
& \gamma_{4}=Z_{2}\left(Z_{2}-Z_{1}\right) /(\Lambda+1) \\
& \gamma_{5}=\left[Z_{2}\left(Z_{2}-Z_{1}\right) s+Z_{1}+1\right] / 2 \\
& \gamma_{6}=Z_{2}\left(Z_{2}-Z_{1}\right) / \Lambda \\
& \gamma_{7}=Z_{2}\left(Z_{2}-Z_{1}\right) s+Z_{1}+1
\end{aligned}
$$


Comparison of the expression (A14), (A12) and their combination with the definition (Al) result in the following:

$$
\begin{aligned}
& \tilde{\boldsymbol{c}}_{\mathbf{3}}(\tilde{\boldsymbol{x}}=0)=\mathrm{M} ; \tilde{\boldsymbol{c}}_{\mathbf{3}}(\tilde{x}=1)=\boldsymbol{M} \omega ; \\
& \tilde{\boldsymbol{c}}_{\mathbf{2}}(\tilde{x}=\mathbf{0})=\boldsymbol{M}(s+\mathrm{N}) ; \tilde{\boldsymbol{c}}_{\mathbf{2}}(\tilde{\boldsymbol{x}}=1)=\boldsymbol{M}\left(s \omega+\mathrm{N} \omega^{\Delta}\right) .
\end{aligned}
$$

Values $\tilde{\boldsymbol{c}}_{1}(\tilde{\boldsymbol{x}}=0)$ and $\tilde{\boldsymbol{c}}_{\mathbf{1}}(\tilde{\boldsymbol{x}}=1)$ are derived from (A16) and (AS). Solving equations (A7), (A8) relatively w,, w, we get:

$$
w_{1}=w_{1}(\omega)=\frac{-\chi^{J}+\left[\left(\chi^{j^{2} 2}+\frac{4 \tilde{c}_{1}(\tilde{x}=0) \tilde{c}_{2}(\tilde{x}=0) Z_{1} Z_{2}}{K(1-K)}\right]^{1 / 2}\right.}{2 \tilde{c}_{2}(\tilde{x}=0) /(1-K)}
$$$$
w_{2}=w_{2}(\omega)
$$

$$
=\frac{-\chi^{J}+\left[\left(\chi^{J}\right)^{2}+\frac{4 \tilde{c}_{2}(\tilde{x}=1) \tilde{c}_{2}(\tilde{x}=1) Z_{1} Z_{2}}{K(1-K)}\right]^{1 / 2}}{2 \tilde{c}_{2}(\tilde{x}=1) /(1-K)} .
$$

(A17)

The overvoltage values $\boldsymbol{\xi}_{\mathbf{L}}$ and $\boldsymbol{\xi}_{\mathbf{R}}$ are determined from the correlation (A17) after replacing of dimensionless variables by dimensional variables:

$$
\begin{aligned}
& \xi_{\mathrm{L}}=\frac{2 R T_{1}}{F\left(z_{1}-z_{2}\right)} \ln w_{\mathrm{I}} \\
& \xi_{\mathrm{R}}=\frac{2 R T_{2}}{F\left(z_{1}-z_{2}\right)} \ln w_{2}
\end{aligned}
$$

As the dimensional potential drop in the transport zone is expressed just as $\boldsymbol{R} \boldsymbol{T} / \boldsymbol{Z}_{\mathbf{3}} \mathrm{Fe}$ ", equation (19) can be used for determination of the potential difference $\boldsymbol{U}=\boldsymbol{U}(\boldsymbol{\omega})$ between the electrodes. Thus, the functions $\boldsymbol{U}(\omega)$ and $\boldsymbol{J}(\omega)$ are determined (see (A14)), and it means that the dependence $J(U)$ is determined parametrically for all possible values of potential difference.

\section{APPENDIX B}

In this appendix the solution of the convective diffusion problem for thermogalvanical cell with vertical arrangement of the electrodes will be given.

Introducing dimensionless concentrations $\tilde{c}_{m}=c_{m} / c_{0}$, it is possible to present equations (29) as

$$
\frac{1}{n} \frac{\partial^{2} \tilde{c}_{m}}{\partial n^{2}}=\theta_{m} \frac{\partial \tilde{c}_{m}}{\partial \tau}, m=1,2
$$

where

$$
\theta_{m}=\frac{\Theta L^{2} h^{2}}{2 D_{m}}, m=1,2
$$

is a dimensionless constant. Due to periodicity of $\tilde{\mathcal{C}}_{m}$ in the segment $(0,2)$, concentrations can be expanded into Fourier series by variable $\tau$; their components $\tilde{c}_{m}^{(1)}$ fulfil the equations:

$$
\begin{gathered}
\frac{1}{n} \frac{\partial^{2} \tilde{c}_{m}^{(l)}}{\partial n^{2}}=\theta_{m} \pi i l \tilde{c}_{m}^{(l)}, l \neq 0 \\
\frac{\partial^{2} \tilde{c}_{m}^{(0)}}{\partial n^{2}}=0, l=0 .
\end{gathered}
$$

Solutions of these equations, restricted at $n \rightarrow \infty$, are the following:

$$
\begin{gathered}
\tilde{c}_{m}^{(l)}=A i\left(\sqrt{\theta_{m} \pi i l} \mathrm{n}\right), \mathrm{I} \neq 0 \\
\tilde{c}_{m}^{(0)}=\tilde{c}_{m}^{0}, \mathrm{I}=0 .
\end{gathered}
$$

Airy function Ai tends to zero exponentially with the argument increase. The complementary Airy function of the second kind Bi increases unlimitedly and must be omitted from solution (B4), The Airy function can be expressed by Bessel functions with index $\frac{1}{3}$, and they are often applied in different problems of mathematical physics, especially in problems of quantum mechanics[20].

Summarising the Fourier series

$$
\tilde{c}_{m}=\tilde{c}_{m}^{0}+\sum_{i=1}^{\infty} F_{m}^{(i)} \mathrm{e}^{i l \pi x} A i\left(\sqrt[3]{\theta_{m} \pi i l} n\right)
$$

we get a solution, satisfying both periodical boundary conditions and initial wnditions for the bulk volume far apart from the electrodes. The indefinite coefficients $\boldsymbol{F}_{\boldsymbol{m}}^{(\boldsymbol{l})}$ should be determined from the boundary wnditions (17), (18) for the electrode surfaces.

As the electrical current on the surface of the electrode is transported only by means of diffusion, we get $j_{1}=$ $z, F D_{1}\left(\partial c_{1} / \partial x\right)$. Taking into account stoichiometric relations for currents (14), (15), boundary conditions (17), (19) can be presented as:

$$
-\left.D_{1} \frac{\partial \tilde{c}_{1}}{\partial n}\right|_{n=0}=\left.D_{2} \frac{\partial \tilde{c}_{2}}{\partial n}\right|_{n=0}=\left.a \tilde{c}_{1}\right|_{n=0}+\left.b \tilde{c}_{2}\right|_{n=0}
$$

where $\mathrm{a}$ and $\mathrm{b}$ are simple step-like functions of coordinate $\tau$ :

$$
\begin{gathered}
a=\left\{\begin{aligned}
\sigma w_{1} / \tilde{c}_{1}^{0} & \text { at } 0<\tau<1 \\
\sigma w_{2} / \tilde{c}_{1}^{0} & \text { at } 1<\tau<2
\end{aligned}\right. \\
b= \begin{cases}-\sigma /\left(w_{1} \tilde{c}_{2}^{0}\right) & \text { at } 0<\tau<1 \\
-\sigma /\left(w_{2} \tilde{c}_{2}^{0}\right) & \text { at } 1<\tau<2\end{cases}
\end{gathered}
$$

and

$$
\begin{array}{r}
\sigma=\frac{j_{0} h}{\tilde{c}^{0}\left(z_{1}-z_{2}\right) F} ; \quad w_{1}=\exp \left(\frac{\left(z_{1}-z_{2}\right) \xi_{\mathrm{L}} F}{2 R T_{l}}\right) \\
w_{2}=\exp \left(\frac{\left(z_{1}-z_{2}\right) \xi_{\mathrm{R}} F}{2 R T_{2}}\right) .
\end{array}
$$

Introducing (B5) into the first equation (B6), multiplying it by $\mathbf{e}^{-i \delta \approx \boldsymbol{x}}$, and integrating in the interval $(0,2)$, we obtain a simple relation for the expansion coefficients.

$$
F_{2}^{(l)}=-D_{2} / D_{1} \sqrt[3]{\theta_{2} / \theta_{1}} F_{1}^{(l)}
$$

and introducing (B6)into the second equation, we get

$$
\begin{aligned}
\sum_{i=1}^{\infty} \mathrm{e}^{i l \pi \pi}\left(\mu \left(a F_{1}^{(l)}\right.\right. & \left.+b F_{2}^{(l)}\right) \\
& \left.+\mu^{\prime} \sqrt[3]{\theta_{1} \pi i l} D_{1} F_{1}^{(l)}\right)=-a \tilde{c}_{1}^{0}-b \bar{c}_{2}^{0} .
\end{aligned}
$$

Here, $\mu=\boldsymbol{A} \dot{\boldsymbol{i}}(\mathbf{0})=0.3550$ and $\boldsymbol{\mu}^{\prime}=\boldsymbol{A} \dot{\boldsymbol{i}}(\mathbf{0})=0.2588$ are constant. Multiplying (B10) by e-'*', $f=1,2, \ldots$ and integrating in the interval $(0,2)$ we get an infinite system of linear equations relatively $F_{1}^{\prime}$.

$$
\sum_{l=1}^{\infty} G_{l f} F_{1}^{(l)}+Q_{f} F_{1}^{(i)}=V_{s}
$$

where

$$
G_{l f}=\left\{\begin{array}{l}
\frac{2 i \mu \sigma}{2 \pi(l-f)}\left(\left(\frac{w_{1}-w_{2}}{c_{1}^{0}}\right)+\frac{D_{2}}{D_{1}} \sqrt[3]{\frac{\theta_{2}}{\theta_{1}}}\right. \\
\left.\quad \times\left(\frac{1}{w_{1} \tilde{c}_{2}^{0}}-\frac{1}{w_{2} \tilde{c}_{2}^{0}}\right)\right) l-f \text {-odd } \\
\mu \sigma\left(\frac{w_{1}+w_{2}}{\tilde{c}_{1}^{0}}+\frac{1}{\tilde{c}_{2}^{0}}\left(\frac{1}{w_{1}}+\frac{1}{w_{2}}\right)\right) \quad l=f \\
0 \quad \text { in other cases }
\end{array}\right.
$$




$$
\begin{gathered}
V_{f}=\left\{\begin{array}{lc}
\frac{2 i \sigma}{f \pi}\left(w_{1}-w_{2}-\frac{1}{w_{1}}-\frac{1}{w_{2}}\right) & f \text {-odd } \\
0 & f-\text { even }
\end{array}\right. \\
Q_{f}=\mu^{\prime} \sqrt[3]{\theta_{1} \pi i f D_{1}} .
\end{gathered}
$$

The system of equations (B11)-(B14) is to be solved relatively $F_{\mathfrak{I}}^{l}$, and after that the solution of (BS) appears to be dependent only on two parameters, $\boldsymbol{w}$, and $\boldsymbol{w}$, . They should be chosen with regard to a condition, that the integral current $\bar{I}$, flowing through the surfaces of two electrodes, is defined by the external source:

$$
\int_{0}^{1} j_{1}(\tau) \mathrm{d} \tau=-\int_{1}^{0} j_{1}(\tau) \mathrm{d} \tau=\frac{\bar{I}}{1-z_{2} / z_{1}} .
$$

So, we get two additional conditions for $F_{1}^{l}$ :

$$
\sum_{s=1}^{\infty} l^{-2 / 3} F_{1}^{(l)}= \pm \frac{\bar{I}}{1-z_{2} / z_{1}} \frac{\tilde{c}_{1}^{0} i \pi}{2 \mu^{\prime} D_{1} F H z_{1} \sqrt[3]{\theta_{1} \pi i}} .
$$

Parameters $\boldsymbol{w}$, and $\boldsymbol{w}$, are to $\boldsymbol{b e}$ found at fixed $\tilde{I}$ from equations (B16), and after that the value of the voltage difference between the electrodes $u$ will be determined from (B8) and
(19). Practically, it is more convenient to use the parametric form of the current-voltage curve with intermediate parameter $\boldsymbol{w}$, and to define the value of the integral current from one of equations (B16). In this case we have to solve numerically only one equation relatively $\boldsymbol{w}$, because the solution of the system (B11) can be obtained analytically with any required accuracy.

There is also the theoretical evaluation of the number of those terms in the system (B11), which are to be taken into account. Actually, the solution in form (B5) is reduced to the expansion of the step-like boundary conditions (B6) by trigonometrical functions. Increasing of the terms number in (BS) leads to the better approximation of the step-like function, but, as a matter of fact, the solution loses its accuracy in the upper and lower regions of the cell, ie in the area of $L / h$ length around the points $\boldsymbol{O}$ and $\mathbf{1}$. It means, that taking into account the terms with their numbers, exceeding

$$
N_{\max }=h / L,
$$

is out of the limits of accuracy. For typical systems $h / L \sim 10$, and the solution of system of equations (B11) does not involve any difficulties. 